\title{
Individual recurrence intervals after anti-VEGF therapy for age-related macular degeneration
}

\author{
Robert Hörster • Tina Ristau • Srinivas R. Sadda • \\ Sandra Liakopoulos
}

Received: 30 July 2010 /Revised: 29 October 2010 /Accepted: 21 November 2010 /Published online: 18 December 2010

(C) The Author(s) 2010. This article is published with open access at Springerlink.com

\begin{abstract}
Background To assess the time interval to recurrent choroidal neovascular membrane $(\mathrm{CNV})$ activity in eyes with neovascular age-related macular degeneration (AMD) after intravitreal anti-VEGF therapy.

Methods Data from all patients who received intravitreal ranibizumab injections for neovascular AMD at the University of Cologne prior to February 2009 were retrospectively reviewed. Patients were treated on a pro re nata (PRN) basis and eyes with active $\mathrm{CNV}$ received three consecutive monthly injections. Recurrence of CNV activity was defined as recurrence of intra- or subretinal fluid on optical coherence tomography (OCT) or leakage on fluorescein angiography (FA) after initial resolution of fluid and leakage following anti-VEGF therapy. All eyes showing at least two documented recurrences of CNV activity during follow-up were included in this analysis. Recurrence intervals were calculated and were deemed to be regular or periodical if the difference between recurrence interval times was less than 50 days.

Results Twenty-nine eyes of 28 patients met the inclusion criteria. Two to six recurrences were detected per case (mean $2.8 \pm 1.1$ recurrences). Recurrence intervals ranged from 41 days to 523 days (mean $5.5 \pm 3.4$ months, median 4.5 months). Twenty-two eyes (76\%) showed at least two
\end{abstract}

R. Hörster · T. Ristau $\cdot$ S. Liakopoulos $(\bowtie)$

Department of Vitreoretinal Surgery, Center for Ophthalmology, University of Cologne,

Kerpener Strasse 62,

50924 Cologne, Germany

e-mail: sandra.liakopoulos@uk-koeln.de

\section{S. R. Sadda}

Doheny Retina Institute, Doheny Eye Institute, Keck School of Medicine of the University of Southern California,

Los Angeles, CA, USA periodical recurrence intervals. In 12 eyes (41\%), all recurrences occurred at regular intervals $(2-4$ recurrences, mean $2.3 \pm 0.6$ recurrences). Seven eyes $(24 \%)$ showed irregular recurrence intervals (2-3 recurrences, mean $2.1 \pm 0.4$ recurrences). All 11 eyes with a classic CNV lesion component showed at least two periodical recurrence intervals. Eyes with occult CNV lesions showed periodical recurrence intervals in 11 out of 18 cases (61\%).

Conclusions Preliminary data indicate that periodical recurrences of CNV activity may be seen in eyes with neovascular AMD undergoing anti-VEGF therapy. Knowledge of individual recurrence interval times may allow for the development of an individualized treatment plan and prophylactic therapy.

Keywords Neovascular age-related macular degeneration . Ranibizumab $\cdot$ Anti-VEGF therapy $\cdot$ Recurrence

\section{Introduction}

Neovascular age-related macular degeneration (AMD) is a leading cause of severe and irreversible vision loss in the developed world among people 50 years of age or older [1-5]. Treatment of neovascular AMD with monthly injections of ranibizumab (Lucentis, Genentech Inc, South San Francisco, CA, USA), an antibody fragment that avidly binds to vascular endothelial growth factor (VEGF), is the standard treatment of care for neovascular AMD [6, 7]. The phase III pivotal clinical trials, Minimally Classic/Occult Trial of the Anti-VEGF Antibody Ranibizumab in the Treatment of Neovascular AMD (MARINA) [7] and Anti-VEGF Antibody for the Treatment of Predominantly Classic Choroidal Neovascularization (CNV) in AMD (ANCHOR) [6], have demonstrated 
the efficacy and safety of monthly ranibizumab injections for the preservation and improvement of visual acuity by using a fixed-dosing regimen requiring a $0.5-\mathrm{mg}$ or $0.3-\mathrm{mg}$ injection of ranibizumab every month for 2 years $[6,7]$.

In the PIER Study [8], patients were given three injections of ranibizumab at monthly intervals followed by injections at a quarterly interval, for a total of six scheduled injections over a 1-year period. Patients showed an early gain in visual acuity after the first three injections, but thereafter had a mean visual acuity decline. Apparently better results were obtained using an optical coherence tomography (OCT) guided, variable dosing regimen (PrONTO study) $[9,10]$. In this small, uncontrolled study, patients were given three consecutive monthly injections of ranibizumab, and were then reassessed monthly. Reinjection was performed if specific pre-defined criteria for recurrent or persistent activity were present, including fluid accumulation on OCT or leakage on fluorescein angiography. Though the PrONTO data is not directly comparable to the phase III trials, PrONTO functional outcomes appeared superior to quarterly injections, though perhaps not as good as with monthly injections. Another prospective study analyzing the outcome after pro re nata (PRN) treatment with bevacizumab (Bashshur) [11, 12], as well as a controlled, prospective phase III study using ranibizumab therapy as needed, the SUSTAIN study, demonstrated similar results [13].

Although the as-needed dosing strategy (PRN treatment) may reduce the number of intravitreal injections and allow the treatment plan to be individualized, it may still require monthly visits to specialized centers. In contrast to mandated monthly injections, patients treated with PRN strategies may develop multiple recurrences of $\mathrm{CNV}$ activity over time. Recurrent intra- or subretinal fluid could potentially induce progressive, cumulative dysfunction of the neural retina, resulting in a decreased ability of the retina to recover despite further treatment.

The "treat-and-extend approach" aims to individualize the treatment plan and decrease the number of injections per year, but at the same time attempts to achieve a fluidfree macula and decrease the number of visits [14-17]. Patients treated with the treat-and-extend regimen typically receive monthly injections until the signs of exudation have resolved. The treatment interval is then sequentially lengthened by 1 to 2 weeks as long as there are no signs of recurrent exudation on OCT or fluorescein angiography (FA). When recurrent exudation is detected on a follow-up visit, the treatment interval is reduced to the prior interval. Treatment is rendered at every visit but the time between visits is individualized based on a given patient's response to treatment.

Another individualized treatment strategy that aims to avoid recurrent $\mathrm{CNV}$ activity as well as additionally to further reduce the number of injections and visits may be to perform the injection immediately prior to the next recurrence. This would require the ability to determine or predict the recurrence interval for an individual patient. In this study, we analyzed the recurrence intervals of patients undergoing anti-VEGF therapy for neovascular AMD to determine whether predictable, regular recurrence patterns were present for individual patients.

\section{Methods}

Data from all patients who began intravitreal ranibizumab therapy for neovascular AMD at the University of Cologne prior to February 2009 were retrospectively reviewed. To be included in this study, eyes were required to demonstrate at least two instances of recurrent $\mathrm{CNV}$ activity during follow-up. Recurrent activity was defined as the re-appearance of fluid on OCT (in the intraretinal or subretinal compartments) and/ or leakage on angiography following a previous fluid-free and/or leakage-free interval. Eyes with persistent fluid requiring continuous injections, eyes with sustained resolution of fluid without two recurrences, and eyes of patients who did not return for follow-up were excluded. Eyes that received photodynamic therapy or vitreous surgery during follow-up were also excluded from this analysis. Best-corrected baseline visual acuity (Snellen), age of the patient, CNV lesion subtype and dates of intravitreal injections were collected. For every visit, OCT and FA images were reviewed to determine whether signs of $\mathrm{CNV}$ activity were present. Approval for data collection and analysis was obtained from the institutional review board of the University of Cologne. The research adhered to the tenets set forth in the Declaration of Helsinki.

In general, patients received three consecutive monthly injections of ranibizumab and were re-treated on a PRN basis. Bevacizumab was used instead of ranibizumab intermittently, if the confirmation for the assumption of costs was delayed by the insurance company. FA was performed in all cases prior to the first injection. At followup visits, FA and/or OCT imaging was performed. Stratus OCT (Carl Zeiss Meditec, Dublin, CA) was used until June 2008, since then a high-resolution Spectral domain OCT (Spectralis HRA + OCT, Heidelberg Engineering GmbH, Dossenheim, Germany) was available. Since 2009, FA was performed at follow-up visits only if CNV activity was questionable based on OCT images, however, at least once a year. Eyes with intra- or subretinal fluid accumulation on OCT or leakage on FA at subsequent follow-up examinations were re-treated with 3 consecutive monthly injections of ranibizumab. If no $\mathrm{CNV}$ activity was detected at follow- 
up, patients were instructed to return immediately if they noticed a decrease in visual acuity or perception of new metamorphopsia, or were scheduled for follow-up after 4-6 weeks.

Recurrence intervals were calculated between the date of the last intravitreal injection and the follow-up visit on which recurrent $\mathrm{CNV}$ activity was diagnosed. For cases in which the difference between recurrence interval times was less than 50 days, the recurrences were deemed to occur at regular intervals, or termed "periodical." If the recurrence interval times differed by more than 50 days, they were termed irregular. The broad 50-day window was chosen as some patients did not return at monthly intervals, and only returned at the first sign of visual decrease or new visual symptoms. Thus, some patients may have had recurrent fluid that preceded their reassessment in the clinic.

Statistical analysis was performed using commercially available software (SigmaPlot 2008 for Windows, version 11.0, SYSTAT Software Inc., Erkrath, Germany). Baseline characteristics were compared between eyes with and without periodical CNV activity using the $t$ test or MannWhitney rank-sum test, depending on whether the data matched the pattern expected in a population with a normal distribution. The Mann-Whitney rank-sum test was also used to compare the recurrence interval times between different CNV lesion types and different anti-VEGF agents. The correlation between CNV lesion subtypes and periodical activity was calculated using Fisher's exact test. The correlation between mean interval duration and baseline visual acuity or age of the patient was calculated using Pearson correlation. $p$ values $<0.05$ were considered statistically significant.

\section{Results}

A total of 646 eyes began ranibizumab therapy for neovascular AMD prior to February 2009 at the University of Cologne. Out of those cases, 390 eyes received fewer than six anti-VEGF injections prior to February 2010, either because no additional injections were required or because they were lost to follow-up at our clinic. Out of the remaining 256 cases, 29 eyes of 28 patients showed two or more documented recurrences of $\mathrm{CNV}$ activity during follow-up and were eligible for analysis. In 11 out of those cases (38\%), bevacizumab was used instead of ranibizumab intermittently. There was no statistically significant difference between recurrence interval times that were calculated following bevacizumab injections and ranibizumab injections.

Ten patients were male $(36 \%)$ and 18 patients female (64\%). Mean age was $75 \pm 7$ years (range 62 to 89 ). Visual acuity at baseline was $0.5 \pm 0.3 \operatorname{logMAR}(20 / 60 \pm 3$ lines, range $20 / 25$ to $20 / 400$ ). Nine cases showed predominantly classic $\mathrm{CNV}$, two cases minimally classic CNV, and 18 eyes occult with no classic CNV. Additionally, retinal angiomatous proliferation (RAP) was present in seven cases, and polypoidal choroidal vasculopathy (PCV) was detected in two cases.

The mean follow-up time was $28.8 \pm 9.2$ months (range from 12 to 42 months). The total number of injections during follow-up ranged from 6 to 19 injections (mean number of injections per year of $4.9 \pm 1.8$ ). Two to 6 recurrences of $\mathrm{CNV}$ activity were detected per case (mean $2.8 \pm 1.1$ recurrences). Recurrence intervals ranged from 41 days to 17 months (mean 5.5 \pm 3.4 months, median 4.5 months) (Fig. 1). There was no significant correlation between the mean or median recurrence interval time and baseline visual acuity or age of the patient.
Fig. 1 Recurrence intervals (months) for all cases. One case showed six recurrences (red), two cases five recurrences (green), three cases four recurrences (blue), seven cases three recurrences (yellow) and 16 cases two recurrences (black and white) during follow-up

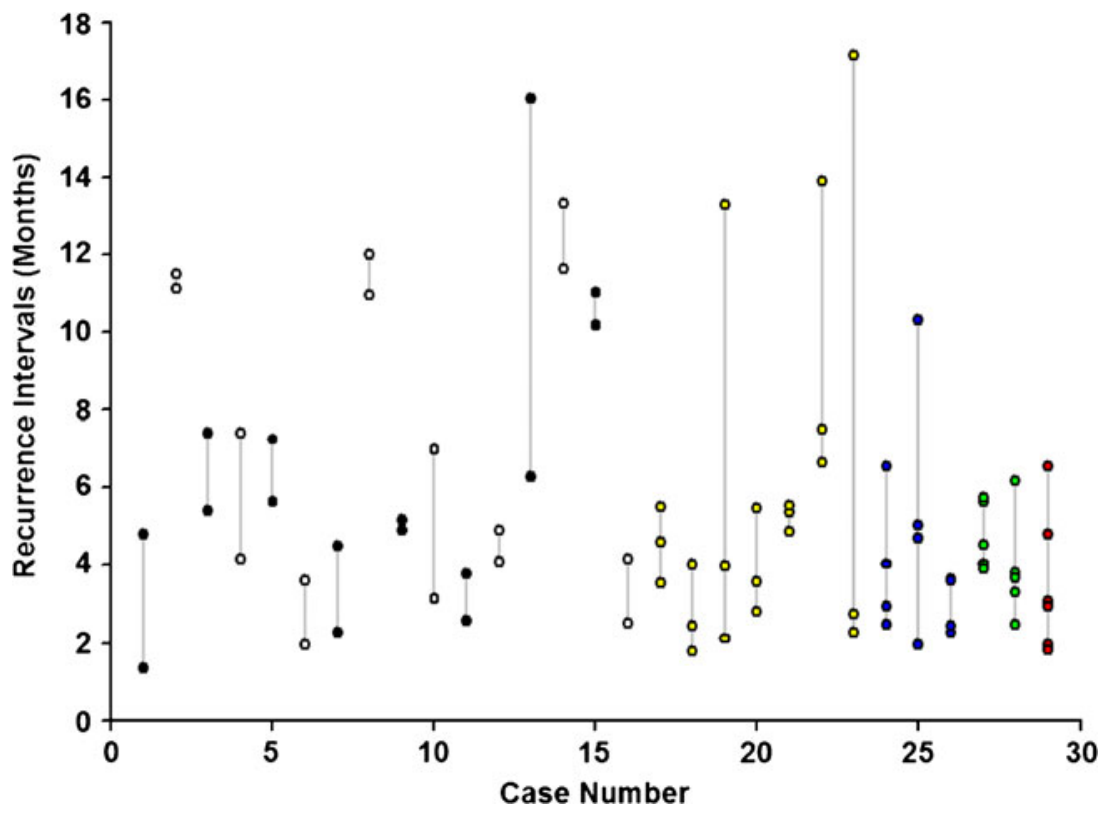


Table 1 Summary of data from all cases

\begin{tabular}{|c|c|c|c|c|c|c|c|c|}
\hline Case no. & $\begin{array}{l}\text { Age at } \\
\text { baseline }\end{array}$ & $\begin{array}{l}\text { Baseline BCVA } \\
\text { (Snellen) }\end{array}$ & $\begin{array}{l}\text { CNV lesion } \\
\text { subtype }\end{array}$ & RAP & $\begin{array}{l}\text { Follow-up } \\
\text { (months) }\end{array}$ & $\begin{array}{l}\text { Number of } \\
\text { recurrences } \\
\text { detected }\end{array}$ & $\begin{array}{l}\text { Periodical } \\
\text { recurrence } \\
\text { intervals }\end{array}$ & $\begin{array}{l}\text { Recurrence intervals times } \\
\text { (Months) }\end{array}$ \\
\hline 1 & 68 & $20 / 30$ & Occult CNV & No & 17.0 & 2 & 0 & $4.8 ; 1.3$ \\
\hline 2 & 77 & $20 / 50$ & Min. classic & No & 33.1 & 2 & 2 & $11.5 ; 11.1$ \\
\hline 3 & 86 & $20 / 80$ & Occult CNV & No & 30.5 & 2 & 0 & $7.4 ; 5.4$ \\
\hline 4 & 87 & $20 / 40$ & Occult CNV & Yes & 21.3 & 2 & 0 & $7.4 ; 4.2$ \\
\hline 5 & 81 & $20 / 25$ & Occult CNV & Yes & 27.7 & 2 & 2 & $5.6 ; 7.2$ \\
\hline 6 & 78 & $20 / 120$ & Pred. classic CNV & No & 18.3 & 2 & 2 & $3.6 ; 1.9$ \\
\hline 7 & 68 & $20 / 25$ & Occult CNV & No & 19.1 & 2 & 0 & $2.3 ; 4.5$ \\
\hline 8 & 63 & $20 / 50$ & Pred. classic CNV & No & 33.0 & 2 & 2 & $12.0 ; 11.0$ \\
\hline 9 & 70 & $20 / 200$ & Pred. classic CNV & No & 17.8 & 2 & 2 & $4.9 ; 5.1$ \\
\hline 10 & 74 & $20 / 40$ & Occult CNV, PCV & No & 25.0 & 2 & 0 & $7.0 ; 3.1$ \\
\hline 11 & 68 & $20 / 160$ & Occult CNV, PCV & No & 14.7 & 2 & 2 & $2.6 ; 3.8$ \\
\hline 12 & 80 & $20 / 50$ & Pred. classic CNV & No & 14.9 & 2 & 2 & $4.1 ; 4.9$ \\
\hline 13 & 77 & $20 / 60$ & Occult $\mathrm{CNV}$ & Yes & 37.6 & 2 & 0 & $16.0 ; 6.3$ \\
\hline 14 & 75 & $20 / 100$ & Pred. classic CNV & No & 33.2 & 2 & 2 & $13.3 ; 11.6$ \\
\hline 15 & 62 & $20 / 25$ & Occult $\mathrm{CNV}$ & Yes & 31.1 & 2 & 2 & $10.2 ; 11.0$ \\
\hline 16 & 82 & $20 / 120$ & Occult CNV & No & 12.1 & 2 & 2 & $4.2 ; 2.5$ \\
\hline 17 & 82 & $20 / 40$ & Pred. classic CNV & No & 35.5 & 3 & 2 & $3.5 ; 4.6 ; 5.5$ \\
\hline 18 & 70 & $20 / 50$ & Occult CNV & No & 33.1 & 3 & 2 & $4.0 ; 2.4 ; 1.8$ \\
\hline 19 & 76 & $20 / 25$ & Occult $\mathrm{CNV}$ & No & 29.8 & 3 & 0 & $13.3 ; 2.1 ; 4.0$ \\
\hline 20 & 71 & $20 / 40$ & Pred. classic CNV & No & 28.5 & 3 & 2 & $3.6 ; 5.5 ; 2.8$ \\
\hline 21 & 71 & $20 / 400$ & Occult CNV & No & 35.5 & 3 & 3 & $5.4 ; 5.5 ; 4.9$ \\
\hline 22 & 76 & $20 / 200$ & Pred. classic CNV & No & 38.6 & 3 & 2 & $7.5 ; 6.7 ; 13.9$ \\
\hline 23 & 77 & $20 / 100$ & Occult $\mathrm{CNV}$ & Yes & 37.6 & 3 & 2 & $17.1 ; 2.3 ; 2.7$ \\
\hline 24 & 79 & $20 / 30$ & Occult CNV & No & 34.9 & 4 & 3 & $6.6 ; 2.5 ; 4.0 ; 2.9$ \\
\hline $25^{\mathrm{a}}$ & 75 & $20 / 50$ & Occult $\mathrm{CNV}$ & Yes & 36.5 & 4 & 2 & $5.0 ; 10.3 ; 4.7 ; 1.9$ \\
\hline 26 & 77 & $20 / 40$ & Min. classic & No & 16.5 & 4 & 4 & $3.6 ; 2.3 ; 3.6 ; 2.4$ \\
\hline $27^{\mathrm{a}}$ & 74 & $20 / 100$ & Occult CNV & Yes & 39.8 & 5 & 3 & $4.5 ; 4.0 ; 5.6 ; 3.9 ; 5.7$ \\
\hline 28 & 89 & $20 / 50$ & Occult CNV & No & 42.7 & 5 & 4 & $3.8 ; 2.5 ; 3.7 ; 6.2 ; 3.3$ \\
\hline 29 & 76 & $20 / 200$ & Pred. classic CNV & No & 41.1 & 6 & 4 & $6.6 ; 3.1 ; 2.9 ; 4.8 ; 1.9 ; 1.8$ \\
\hline
\end{tabular}

${ }^{\mathrm{a}}$ Both eyes of one patient

Abbr.: $C N V$ choroidal neovascularization, Min. minimal, Pred. predominantly, $B C V A$ best-corrected visual acuity, $R A P$ retinal angiomatous proliferation, $P C V$ polypoidal choroidal vasculopathy. For cases in which the difference between recurrence interval times was less than 50 days, the recurrences were termed "periodical"

Twenty-two out of the 29 eyes (76\%) showed a periodical activity of at least two recurrence intervals, with 12 eyes (41\% out of 29$)$ showing periodical activity of all recurrence intervals (number of recurrences in this group ranged from 2 to 4 , mean $2.3 \pm 0.6$ recurrences). Seven eyes (24\%) showed irregular recurrences (range 2-3 recurrences, mean $2.1 \pm 0.4$ recurrences). A summary of all data is provided in Table 1.

There was a statistically significant difference $(p<0.05)$ regarding baseline visual acuity between eyes with no periodical recurrence intervals (mean 20/40 \pm 2 lines) and eyes with at least two periodical recurrence intervals (mean $20 / 80 \pm 3$ lines). However, there was no statistically significant difference between the two groups regarding the age of the patient or the mean or median recurrence interval times. For example, one eye developed two regular CNV recurrences 5 months after the last injection that was required to resolve all intra- or subretinal fluid, whereas two eyes showed CNV recurrences after 11 and 12 months. One patient presented with four recurrences of $\mathrm{CNV}$ activity within a follow-up time of 16.5 months, all detected 2.3 to 3.6 months after the last injection. One patient demonstrated six recurrences of CNV activity during a follow-up of 41 months, all within the range of 1.8 to 6.6 months after the last injection. Four out of these six recurrences were detected within the range of 1.8 3.1 months and were considered regular. In one patient, both eyes were eligible for analysis. Both eyes demonstrated occult CNV with RAP, one eye developed five recurrences within a follow-up time of 40 months that occurred 3.9, 4.0, 4.5, 5.6, and 5.7 months after the last injection (Fig. 2). Three out of these five recurrences were 


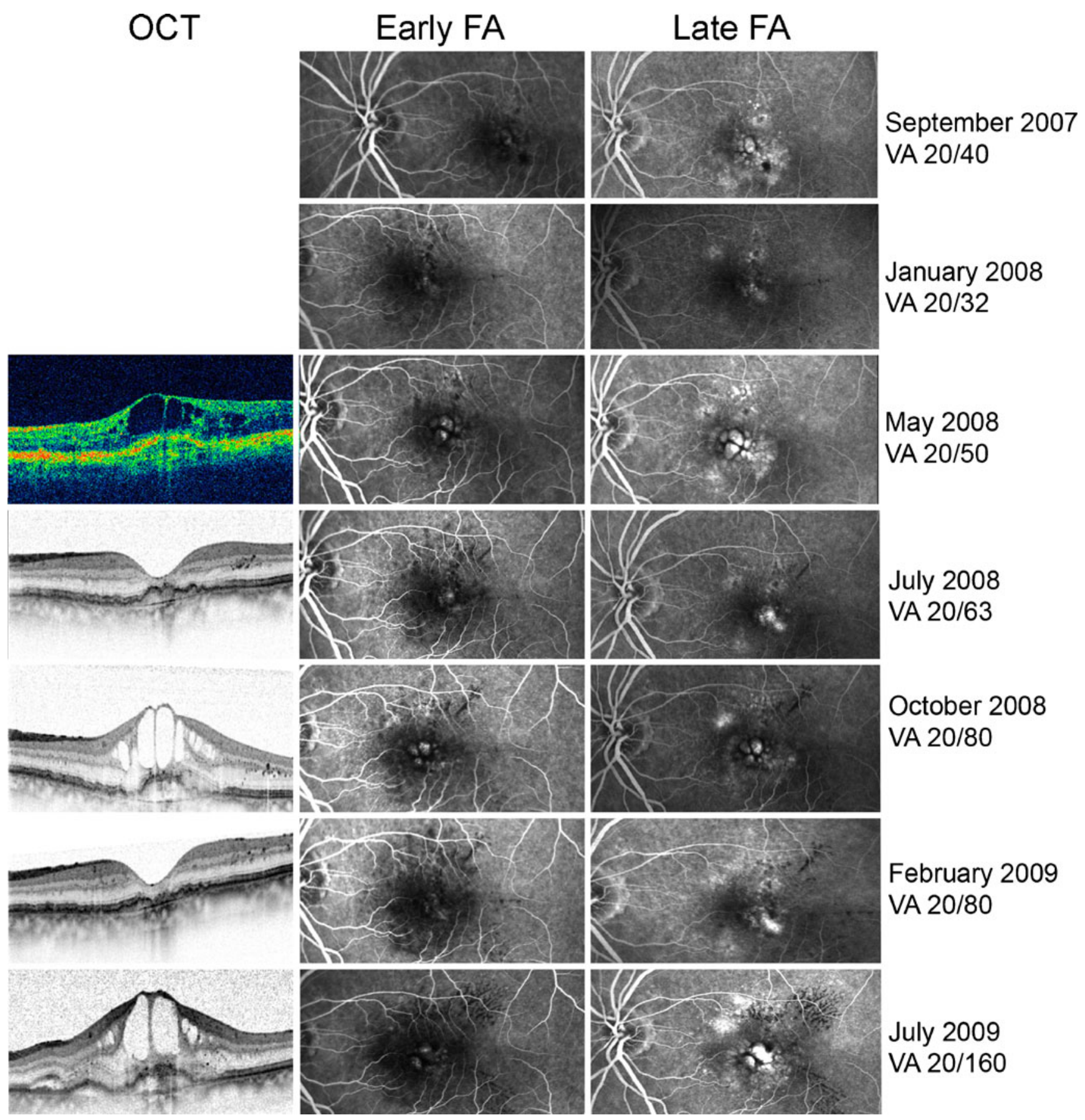

Fig. 2 Example showing a case with occult $\mathrm{CNV}$ and RAP demonstrating five recurrences during a 3-year follow-up (the most recent three out of those five are shown here). The recurrence interval times ranged between 3.9 and 5.7 months. Visual acuity (VA) decreased progressively over time. $O C T$ Optical coherence tomography, $F A$ fluorescein angiography considered periodical, the maximum difference between intervals was 56 days. The fellow-eye presented four recurrences within a follow-up of 37 months (recurrence intervals $1.9,4.7,5.0$, and 10.3 months, two out of those four were considered periodical).

The CNV lesion type for each eye was defined using FA at baseline. All eyes with classic CNV lesion components (predominantly classic and minimally classic $\mathrm{CNV}$ ) showed at least two periodical recurrence intervals (11 out of 11 cases), whereas eyes with purely occult $\mathrm{CNV}$ lesions showed periodical recurrence intervals only in 11 out of 18 cases $(61 \%$, including five out of seven eyes with RAP) $(p=0.026)$ (Fig. 3). However, there was no statistically significant difference between $\mathrm{CNV}$ lesion 


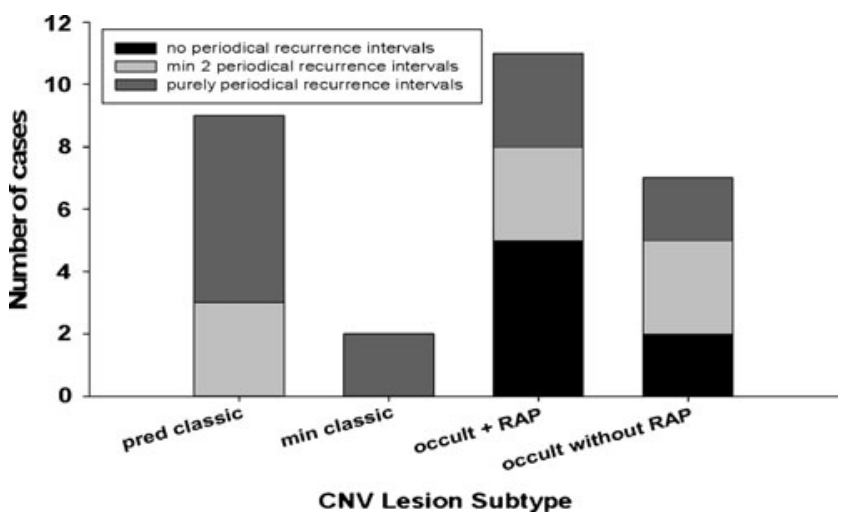

Fig. 3 Frequency of periodical recurrence interval times in various CNV lesion types

types regarding the mean or median recurrence interval time.

\section{Discussion}

Determining the optimal dosing schedule for anti-VEGF therapy in neovascular AMD remains a challenge because neither the effect of the drugs in human eyes nor the nature of the disease itself is fully understood. The MARINA and ANCHOR trials so far reported the best functional outcome results by using monthly injections of ranibizumab [6, 7]. Monthly injections may be necessary in some patients in order to achieve the best possible increase in visual acuity and to preserve retinal function over time. However, many patients may require fewer injections. PRN treatment regimens as well as the treat and extend regimen aim to decrease the number of injections per year. In our dataset, eyes treated "as needed" received a mean number of $4.9 \pm$ 1.8 injections per year. Prospective studies using a PRN treatment regime also reported a lower injection rate per year (PrONTO [9]: 5.6 \pm 2.3 injections per year, Bashshur [11]: 3.4 injections per year). The PrONTO results showed a great inter-patient variability in the number of injections needed, ranging from 3 to 23 throughout a 2-year period [10]. In addition, the PrONTO study was the first to show that in certain specific cases, treatment by ranibizumab could be stopped, at least temporarily, without any loss of benefit. No statistically significant correlation was found between the need for more frequent injections and visual acuity outcomes in the PrONTO and in the Bashshur study [9-12].

However, PRN treatment so far failed to demonstrate functional outcomes comparable to the MARINA and ANCHOR results. Additionally, patients treated less than monthly may develop multiple recurrences of CNV activity, potentially resulting in progressive damage of the neurosensory retina and compromising the long-term visual outcome [15]. In the PrONTO study, 37 out of 40 eyes (93\%) developed recurrent fluid within the 12-month follow-up period [9]. Dagostar et al. reported recurrences of CNV activity in 62 out of 131 eyes $(47.3 \%)$ treated with ranibizumab as needed with a follow-up of at least 6 months (mean 12 months) in a retrospective study [18]. Our dataset does not provide information regarding the incidence of $\mathrm{CNV}$ recurrences, as we included only eyes with at least two documented recurrences in our analysis. Despite further treatment, recovery of visual acuity may be less likely after frequent recurrence of fluid than in treatment naive eyes.

The "treat-and-extend approach" aims to achieve a fluid-free macula and decrease the number of visits using an individualized treatment plan with intervals that are sequentially lengthened until recurrent CNV activity is detected [14-17]. Gupta et al. reported that in $45.7 \%$ of eyes, no recurrent $\mathrm{CNV}$ activity was detected during a mean follow-up period of 1.52 years [14]. However, $46.7 \%$ of eyes still showed between 1 and 4 recurrences (the remaining $7.6 \%$ of eyes demonstrated persistent fluid despite monthly treatment). Eyes treated with the treatand-extend regimen receive injections at every visit. Thus, the reported mean number of 8.36 injections in the first year and 7.45 injections in the second year was higher than reported in studies using the PRN approach. Engelbert et al. used the treat-and-extend approach for 11 eyes with retinal angiomatous proliferation and reported a total of 21 recurrences during a cumulative observation period of 336 months [17]. The mean number of injections was seven in the first year, six in the second year, and seven in the third year.

It is desirable to find a dosing regimen that is maximally effective at preserving or improving visual acuity while at the same time is intended to avoid CNV recurrences as well as to further decrease the number of visits and injections when they are not needed. One possible approach that may fulfill those conditions would be to determine the individual time point of every $\mathrm{CNV}$ recurrence. Re-injections of ranibizumab shortly prior to a recurrence may avoid recurrent leakage and fluid accumulation, as well as further growth of the CNV lesion size. Therefore, avoiding recurrent $\mathrm{CNV}$ activity with prophylactic injections may protect the neural retina from additional damage and improve the long-term prognosis. However, this approach requires the ability to determine individual recurrence intervals. Our study aims to analyze recurrence intervals detected using a PRN regimen, and to determine whether periodical recurrences may exist.

In our dataset, 22 out of 29 eyes demonstrated at least two recurrences of $\mathrm{CNV}$ activity during follow-up that occurred within a similar time interval ( \pm 50 days). Some patients developed multiple regular recurrences, e.g., in one case, four recurrences ranging between 2.3 and 3.6 months 
were detected. The recurrence interval times in our study ranged from 41 days to 17 months, the mean recurrence interval time was $5.5 \pm 3.4$ months, the median 4.5 months (calculated between the last injection that was necessary to resolve all intra- or subretinal fluid and the date the recurrence was noted). There was no difference regarding the mean or median recurrence interval time between eyes with regular recurrence intervals and eyes with irregular recurrence intervals, indicating that eyes that develop a recurrence of $\mathrm{CNV}$ activity early after the injection, are not more likely to show periodical recurrence intervals than eyes that remain fluid-free for months. Some eyes showed regular recurrences after 5 months, some after 11 or 12 months. No correlation could be observed between baseline visual acuity or age of the patient, and recurrence interval times. The PrONTO study reported a mean injection-free interval time of $4.5 \pm 2.7$ months, ranging between 2 and 10 months $[9,10]$. Gupta et al., using the treat-and-extend approach reported a mean maximum period of extension of 79.9 days. No information is provided in both studies regarding inter- or intra-individual variability of recurrence intervals.

Our dataset does not allow to determine whether the differences in recurrence interval times represent differences between individuals (e.g., genetically determined) or between eyes (e.g., due to various CNV lesion types or duration of the disease), as there was only one case with bilateral recurrences in our study. In this case, one eye demonstrated five recurrences (detected after 3.9, 4.0, 4.5, 5.6, and 5.7 months), the fellow eye demonstrated four recurrences (detected after 1.9, 4.7, 5.0, and 10.3 months). Five out of these nine recurrences were considered periodical (difference less than 50 days), seven out of these nine recurrences of both eyes were detected between 119 and 175 days after the last injection (thus within a time interval of 56 days).

Our study was not designed to determine whether $\mathrm{CNV}$ lesion type influenced the risk to develop a recurrence of $\mathrm{CNV}$ activity. However, out of those cases in which more than two recurrences were detected, eyes with a classic $\mathrm{CNV}$ lesion component appeared to be more likely to manifest a regular recurrence interval than eyes with occult $\mathrm{CNV}$ only. Because classic $\mathrm{CNV}$ is believed to correspond to type II histologic CNV (i.e., in the subretinal space), it is possible that patients whose eyes contained classic $\mathrm{CNV}$ may be more sensitive to detecting early signs of recurrence than patients with occult CNV (type I histologic CNV in the sub-RPE space). Certainly, it is well established that classic $\mathrm{CNV}$ lesions are often associated with a more rapid decrease in vision, whereas occult CNV lesions may remain indolent for prolonged periods of time [19]. We also observed that eyes with at least two periodical recurrence intervals presented with poorer baseline visual acuity than eyes with irregular recurrences, but the explanation for this phenomenon is uncertain.

Limitations of our study include the retrospective collection of the data, as well as the small dataset. As we did not monitor all patients monthly, one cannot exclude the possibility that recurrences of CNV activity have been detected somewhat later because patients did not perceive the decrease in vision or delayed the examination. To address this, differences in recurrence interval times up to 50 days were still considered regular. Only 29 cases were eligible for analysis out of 646 eyes that received ranibizumab therapy within the analyzed time period. This may be explained by the fact that most cases only received few injections in Cologne but were followed and re-injected by outpatient doctors. Some patients may have been treated until the intra- and subretinal fluid resolved and stayed dry thereafter, some never showed complete absence of fluid, and thus did by definition not suffer from a recurrence. Furthermore, some patients may have suffered from a severe decrease in vision due to exudative process and may have been lost to follow-up.

Despite those limitations, we could demonstrate that regular recurrences of $\mathrm{CNV}$ activity may occur in some eyes with neovascular AMD, and the recurrence interval time may vary between individuals. The reason for this is not yet fully understood. A larger, prospective trial would be required to evaluate whether individually adjusted prophylactic injections of ranibizumab may allow avoidance of $\mathrm{CNV}$ recurrences, while still reducing the number of injections and visits per year and ultimately improving the long-term outcome in patients with neovascular AMD.

\section{Financial Interest None.}

Open Access This article is distributed under the terms of the Creative Commons Attribution Noncommercial License which permits any noncommercial use, distribution, and reproduction in any medium, provided the original author(s) and source are credited.

\section{References}

1. Augood C, Fletcher A, Bentham G, Chakravarthy U, de Jong PT, Rahu M, Seland J, Soubrane G, Tomazzoli L, Topouzis F, Vioque J, Young I (2004) Methods for a population-based study of the prevalence of and risk factors for age-related maculopathy and macular degeneration in elderly European populations: the EUREYE study. Ophthalmic Epidemiol 11:117-129

2. Bressler NM (2004) Age-related macular degeneration is the leading cause of blindness. JAMA 291:1900-1901

3. Friedman DS, O'Colmain BJ, Munoz B, Tomany SC, McCarty C, de Jong PT, Nemesure B, Mitchell P, Kempen J (2004) Prevalence 
of age-related macular degeneration in the United States. Arch Ophthalmol 122:564-572

4. Resnikoff S, Pascolini D, Etya'ale D, Kocur I, Pararajasegaram R, Pokharel GP, Mariotti SP (2004) Global data on visual impairment in the year 2002. Bull World Health Organ 82:844851

5. Ferris FL 3rd, Fine SL, Hyman L (1984) Age-related macular degeneration and blindness due to neovascular maculopathy. Arch Ophthalmol 102:1640-1642

6. Brown DM, Kaiser PK, Michels M, Soubrane G, Heier JS, Kim RY, Sy JP, Schneider S (2006) Ranibizumab versus verteporfin for neovascular age-related macular degeneration. N Engl J Med 355:1432-1444

7. Rosenfeld PJ, Brown DM, Heier JS, Boyer DS, Kaiser PK, Chung CY, Kim RY (2006) Ranibizumab for neovascular age-related macular degeneration. N Engl J Med 355:1419-1431

8. Regillo CD, Brown DM, Abraham P, Yue H, Ianchulev T, Schneider S, Shams N (2008) Randomized, double-masked, sham-controlled trial of ranibizumab for neovascular age-related macular degeneration: PIER Study year 1. Am J Ophthalmol $145: 239-248$

9. Fung AE, Lalwani GA, Rosenfeld PJ, Dubovy SR, Michels S, Feuer WJ, Puliafito CA, Davis JL, Flynn HW Jr, Esquiabro M (2007) An optical coherence tomography-guided, variable dosing regimen with intravitreal ranibizumab (Lucentis) for neovascular age-related macular degeneration. Am J Ophthalmol 143:566583

10. Lalwani GA, Rosenfeld PJ, Fung AE, Dubovy SR, Michels S, Feuer W, Davis JL, Flynn HW Jr, Esquiabro M (2009) A variabledosing regimen with intravitreal ranibizumab for neovascular agerelated macular degeneration: year 2 of the PrONTO Study. Am J Ophthalmol 148(43-58):e41
11. Bashshur ZF, Haddad ZA, Schakal A, Jaafar RF, Saab M, Noureddin BN (2008) Intravitreal bevacizumab for treatment of neovascular age-related macular degeneration: a one-year prospective study. Am J Ophthalmol 145:249-256

12. Bashshur ZF, Haddad ZA, Schakal AR, Jaafar RF, Saad A, Noureddin BN (2009) Intravitreal bevacizumab for treatment of neovascular age-related macular degeneration: the second year of a prospective study. Am J Ophthalmol 148(59-65):e51

13. Meyer CH, Eter N, Holz FG (2008) Ranibizumab in patients with subfoveal choroidal neovascularization secondary to age-related macular degeneration. Interim results from the sustain trial [abstract]. Invest Ophthalmol Vis Sci: E-abstract 273

14. Gupta OP, Shienbaum G, Patel AH, Fecarotta C, Kaiser RS, Regillo CD (2010) A treat-and-extend regimen using ranibizumab for neovascular age-related macular degeneration clinical and economic impact. Ophthalmology. Jun 29 (epub ahead of print)

15. Brown DM, Regillo CD (2007) Anti-VEGF agents in the treatment of neovascular age-related macular degeneration: applying clinical trial results to the treatment of everyday patients. Am J Ophthalmol 144:627-637

16. Spaide R (2007) Ranibizumab according to need: a treatment for age-related macular degeneration. Am J Ophthalmol 143:679-680

17. Engelbert M, Zweifel SA, Freund KB (2009) "Treat and extend" dosing of intravitreal antivascular endothelial growth factor therapy for type 3 neovascularization/retinal angiomatous proliferation. Retina 29:1424-1431

18. Dadgostar H, Ventura AA, Chung JY, Sharma S, Kaiser PK (2009) Evaluation of injection frequency and visual acuity outcomes for ranibizumab monotherapy in exudative age-related macular degeneration. Ophthalmology 116:1740-1747

19. Pauleikhoff D (2005) Neovascular age-related macular degeneration: Natural history and treatment outcomes. Retina 25:1065-1084 